\title{
Renormalization persistency of tensor force in nuclei
}

\author{
Naofumi Tsunoda \\ Department of physics, the University of Tokyo, 7-3-1 Hongo, Bunkyo-ku, Tokyo, Japan \\ Takaharu Otsuka \\ Department of physics and Center for Nuclear Study, \\ the University of Tokyo, 7-3-1 Hongo, Bunkyo-ku, Tokyo, Japan \\ National Superconducting Cyclotron Laboratory, Michigan State University, East Lansing, MI, 48824, USA \\ Koshiroh Tsukiyama \\ Department of physics and Center for Nuclear Study, \\ the University of Tokyo, 7-3-1 Hongo, Bunkyo-ku, Tokyo, Japan \\ Morten Hjorth-Jensen \\ Department of Physics and Center of Mathematics for Applications, University of Oslo, N-0316 Oslo, Norway \\ National Superconducting Cyclotron Laboratory, Michigan State University, East Lansing, MI, 48824, USA
}

\begin{abstract}
In this work we analyze the tensor-force component of effective interactions appropriate for nuclear shell-model studies, with particular emphasis on the monopole term of the interactions. Standard nucleon-nucleon $(N N)$ interactions such as AV8' and $\chi \mathrm{N}^{3} \mathrm{LO}$ are tailored to shell-model studies by employing $V_{\text {lowk }}$ techniques to handle the short-range repulsion of the $N N$ interactions and by applying many-body perturbation theory to incorporate in-medium effects. We show, via numerical studies of effective interactions for the $s d$ and the $p f$ shells, that the tensor-force contribution to the monopole term of the effective interaction is barely changed by these renormalization procedures, resulting in almost the same monopole term as the one of the bare $N N$ interactions. We propose to call this feature Renormalization Persistency of the tensor force, as it is a remarkable property of the renormalization and should have many interesting consequences in nuclear systems. For higher multipole terms, this feature is maintained to a somewhat smaller extent. We present general intuitive explanations for the Renormalization Persistency of the tensor force, as well as analyses of core-polarization terms in perturbation theory. The central force does not exhibit a similar Renormalization Persistency.
\end{abstract}

PACS numbers: 21.30.Fe, 21.60.Cs

Keywords: tensor force, effective interaction

\section{INTRODUCTION}

The nucleon-nucleon (NN) interaction is normally modelled in terms of several components, such as a central force, a spin-orbit force or a tensor force. These mathematical terms accommodate our phenomenological knowledge of the strong interaction, which, when used in a nuclear many-body context is subjected to different renormalization procedures. For the nuclear many-body problem, a given renormalization procedure leads to the derivation of an effective interaction, starting from a bare realistic NN interaction. The so-called bare NN interactions exhibit a strong coupling between low-momentum and high-momentum degrees of freedom generated from short-range details of the interaction. By "bare" we mean that the abovementioned strong coupling is left untouched. This coupling is included only implicitly, via various renormalization procedures, in the effective interactions used in for example shell-model studies.

As an example of bare NN interactions, the Argonne interactions (AV), which are defined in terms of local operators in coordinate space, show a strong short-range repulsion [1, 2]. The resulting strong coupling between low- and high-momentum modes makes the many-body problem highly non-perturbative. On the other hand, in shell-model calculations, the employed effective interactions are defined for a specific configuration space (a strongly reduced Hilbert space), normally called the model space. Therefore, the effective interactions for the shell model should be renormalized to include the effects of virtual excitations to the configurations not included in the model space.

Although the properties and the effects of the full interaction and various renormalized interactions have been investigated extensively over the years, we feel that there are still important features of the nuclear interaction which deserve some special attention. In particular, we show here via several numerical studies, that the tensor force component of the bare nuclear interaction is left almost unaffected by various renormalization procedures. The monopole component of the tensor force, a component of great interest in studies of shell evolution (see discussion below) in nuclei toward the drip lines, is left almost unchanged under various renormalizations. This allows us thereby to extract simple physics interpretations from complicated many-body systems. In this work we label such a lack of renormalization influence as Renor- 
malization Persistency, in short just R-Persistency. The R-Persistency is a property exhibited by specific terms of the original nuclear Hamiltonian that are not affected, or barely affected, by the renormalization procedure.

On the experimental side, present and future radioactive ion-beam facilities have made it possible to perform experiments that explore nuclei far from the stability line of the nuclear chart. Many unexpected and new phenomena have been observed in such experiments carried out at radioactive ion-beam facilities worldwide. One of the most striking results is the breaking of the conventional shell structures in neutron-rich nuclei. Such shell evolution, unexpected in the past, is known by now to occur mainly due to an unbalanced neutron to proton ratio and specific rbital-dependent components of the nuclear forces. In particular, the nuclear tensor force plays a key role here, as proposed by one of the authors [3, 4]. One of the most useful quantities to probe the effect of tensor force is the so-called monopole matrix element. The monopole matrix element [5, 6] of the two-body interaction between two single-particle states labelled $j$ and $j^{\prime}$ and total two-particle isospin $T$ is defined as

$$
V_{j, j^{\prime}}^{T}=\frac{\sum_{J}(2 J+1)\left\langle j j^{\prime}|V| j j^{\prime}\right\rangle_{J T}}{\sum_{J}(2 J+1)},
$$

for $j \neq j^{\prime}[20]$. Here $\langle\cdot \cdot|V| \cdot \cdot\rangle_{J T}$ denotes the antisymmetrized two-body matrix element coupled to total angular momentum $J$ and total isospin $T$. The monopole matrix element is crucial for shell evolution, because it affects the effective single particle energy linearly. For instance, if $n_{n}\left(j^{\prime}\right)$ neutrons occupy the single-particle state $j^{\prime}$, they shift the effective single particle energy of protons in the state $j$ as follows,

$$
\Delta \epsilon_{p}(j)=\frac{1}{2}\left(V_{j j^{\prime}}^{T=0}+V_{j j^{\prime}}^{T=1}\right) n_{n}\left(j^{\prime}\right),
$$

where $\Delta \epsilon_{p}(j)$ represents the change of the effective single particle energy of protons in the single-particle state $j$. When we consider the tensor-force contribution, the monopole matrix elements always have different signs between a pair of spin-orbit partners. For example, the interaction matrix elements $V_{j>j^{\prime}}$ and $V_{j_{<j^{\prime}}}$ have opposite sign. Here we define $j_{>}$and $j_{<}$to represent the spin-orbit partners, that is, $j_{>}=l+1 / 2$ and $j_{<}=l-1 / 2$, where $l$ stands for the orbital momentum of a given singleparticle state. In this case, the tensor-force changes the spin-orbit splitting between $j_{>}$and $j_{<}$. The shell structure is also altered, in particular if we have a sizable number of neutrons in the single-particle state $j^{\prime}$.

In previous studies [3], the tensor-force component in effective interactions for shell-model calculations was, for the sake of simplicity, modelled via the exchange of $\pi$ and $\rho$ mesons only. To a large extent, this yields results close to the tensor force in realistic NN interactions. In fact, this model describes rather well the experimental data in several mass regions [4]. However, it is far from trivial that the tensor force in effective interactions for the shell- model can be considered to be given by the exchange of $\pi$ and $\rho$ mesons only.

The aim of this article is thus to investigate the RPersistency of the nuclear tensor force and understand the validity of the above assumption through theoretical studies, based on realistic NN interactions and microscopic theories for deriving effective interactions, focusing on the effective interaction for the shell model [21].

This work is organized as follows. First we briefly review the theory for constructing effective interactions in Sec. III In Sec. III and Sec. V] the R-Persistency of the monopole part of the tensor force from various approaches to the effective interactions will be discussed. In Sec. VI we present not only the monopole part but also the two-body matrix elements including multipole part of the various effective interactions and discuss their tensor force components. For the sake of completeness, we include analyses using other NN interactions in Sec. VII. The last section contains our conclusions.

\section{CONSTRUCTION OF THE EFFECTIVE INTERACTION FOR THE SHELL MODEL}

The aim of this section is to give a brief sketch of the theoretical methods we employ in our analyses of the nuclear force. To construct the effective interactions for the nuclear shell model, we use many-body perturbation theory (MBPT). However, as inputs to MBPT, we cannot use bare realistic NN interactions directly, since their high-momentum components make MBPT nonconvergent [7]. We integrate out this high-momentum components employing a renormalized interaction defined only in the low-momentum space below a certain sharp cutoff $\Lambda$ and designed not to change two-body observables like NN scattering data [8]. This recipe defines a cutoff dependent family of interactions, normally labelled as $V_{\text {lowk}}(\Lambda)$, which to be more specific, can be written as

$$
V_{\text {low } k}(\Lambda)=P_{\Lambda} V_{\text {bare }} P_{\Lambda}+\delta V_{c t}(\Lambda),
$$

where $P_{\Lambda}$ indicates a projection operator onto the lowmomentum space below $\Lambda$. The term $\delta V_{c t}(\Lambda)$ represents the correction term coming from the renormalization procedure. In other words, $P_{\Lambda} V_{\text {bare }} P_{\Lambda}$ is a simple projection to a low-momentum space, while $\delta V_{c t}(\Lambda)$ emerges as a result of the chosen renormalization procedure. By construction, $V_{\text {low } k}(\Lambda)$ approaches the original $\mathrm{NN}$ interaction in the limit $\Lambda \rightarrow \infty$. A complete renormalization scheme would generate higher-body forces as well, such as three-body and four-body forces, $V_{3 \mathrm{~N}}$ and $V_{4 \mathrm{~N}}$, respectively. In this work we limit ourselves to two-body $\left(V_{2 \mathrm{~N}}\right)$ interactions only. Thus the cutoff dependence of physical quantities can be used to assess the error made by omitting more complicated many-body forces. The term $P_{\Lambda} V_{\text {bare }} P_{\Lambda}$ should contain the long-range part of one-pion exchange interaction as a major component. 
Next, we proceed to MBPT. The low-momentum interaction $V_{\text {lowk }}$ is a good starting point for MBPT because we can avoid the difficulty caused by the strong short-range repulsion. For a degenerate model space, the effective interaction $V_{\text {eff }}$ can be written as

$$
V_{\mathrm{eff}}=\hat{Q}-\hat{Q}^{\prime} \int \hat{Q}+\hat{Q}^{\prime} \int \hat{Q} \int \hat{Q}-\cdots,
$$

where $\hat{Q}\left(E_{0}\right)$ is the so-called $\hat{Q}$-box, defined as

$$
\hat{Q}\left(E_{0}\right) \equiv P H_{1} P+P H_{1} Q \frac{1}{E_{0}-Q H Q} Q H_{1} P .
$$

Here, the Hamiltonian is divided into an unperturbed part $H_{0}$ and an interaction part $H_{1}, H=H_{0}+H_{1}$ and the model space is set to be degenerate with respect to the unperturbed Hamiltonian $H_{0}$ with energy $E_{0}$. The integration symbols in Eq. (4) represent the inclusion to infinite order of so-called folded diagrams, see Refs. [9, 10] for details. The $\hat{Q}$-box is given by diagrams which are valence-linked and irreducible, while $\hat{Q}^{\prime}$ indicates that only diagrams which are of second- or higher-order in terms of the interaction $H_{1}$, are included, see for example Fig. 1

We can solve Eq. (4) by the following iterative formula

$$
V_{\mathrm{eff}}^{(n)}=\hat{Q}\left(E_{0}\right)+\sum_{m=1}^{\infty} \hat{Q}_{m}\left(E_{0}\right)\left\{V_{\mathrm{eff}}^{(n-1)}\right\}^{m}
$$

where $\hat{Q}_{m}\left(E_{0}\right)=\frac{1}{m !}\left(\frac{\mathrm{d}^{m} \hat{Q}(\omega)}{\mathrm{d} \omega^{m}}\right)_{\omega=E_{0}}$. In this work, we take into account diagrams up to second or third order in the interaction $H_{1}$ for the calculation of the $\hat{Q}$-box.

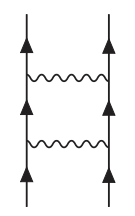

(a)

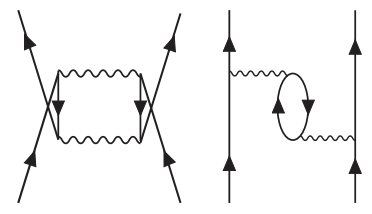

(c)
FIG. 1: Examples of diagrams to second-order in the interaction $H_{1}$ included in the $\hat{Q}$-box. The diagrams are referred to as (a) particle-particle ladder, (b) hole-hole ladder and (c) core-polarization, respectively.

By using this two-step method, we can start from an arbitrary bare realistic NN interaction. We calculate effective interactions starting from AV8' 1, 2] and the chiral $\chi \mathrm{N}^{3} \mathrm{LO}$ interaction 11]. Results using the AV8' interaction are shown in the following sections while our results obtained with the $\chi \mathrm{N}^{3} \mathrm{LO}$ interaction are shown in Sec. VII for the sake of completeness.

Finally, to extract the tensor component from the obtained effective interactions, we employ the spin-tensor decomposition employed in for example Refs. [12 14

$$
\begin{aligned}
& \left\langle a b L S\left|V_{p}\right| c d L^{\prime} S^{\prime}\right\rangle_{J^{\prime} T}=(-1)^{J^{\prime}} \hat{p}\left\{\begin{array}{ccc}
L & S & J^{\prime} \\
S^{\prime} & L^{\prime} & p
\end{array}\right\} \\
& \times \sum_{J}(-1)^{J} \hat{J}\left\{\begin{array}{ccc}
L & S & J \\
S^{\prime} & L^{\prime} & p
\end{array}\right\}\left\langle a b L S|V| c d L^{\prime} S^{\prime}\right\rangle_{J T},
\end{aligned}
$$

where $\left\langle\cdots L S|V| \cdots L^{\prime} S^{\prime}\right\rangle_{J T}$ denotes the $L S$-coupled matrix element of the effective interaction. Here $a$ (as well as $b c d)$ is shorthand for the set of quantum numbers $\left(n_{a}, l_{a}\right)$, etc. The operator $V_{p}$ is defined as the scalar product $V_{p} \equiv U^{(p)} \cdot X^{(p)}$, where $U^{(p)}$ and $X^{(p)}$ are irreducible tensors of rank $p$, applying to operators in both spin and coordinate space. The tensor component is extracted by setting $p=2$ in Eq. (7). Finally, in the above equation we have defined $\hat{p}=2 p+1$ and $\hat{J}=2 J+1$.

\section{TENSOR FORCE IN LOW-MOMENTUM INTERACTION $V_{\text {low } k}$}

We now present results obtained by the theoretical methods described in the previous section. Figure 2 shows the monopole part of the tensor-force of the renormalized $V_{\text {lowk }}$ interaction derived from the Argonne V8' (AV8') potential for the $s d$-shell and the $p f$-shell. The cutoff value $\Lambda$ varies from $1.0 \mathrm{fm}^{-1}$ to $5.0 \mathrm{fm}^{-1}$. Here, we employ units where $c=\hbar=\hbar^{2} / m=1$. The typical value of the cutoff is determined by the best reproduction of the binding energies of ${ }^{3} \mathrm{H}$ and ${ }^{4} \mathrm{He}$. The resulting cutoff value lies around $2.0 \mathrm{fm}^{-1}[15]$. A too small cutoff $\Lambda$ (for example $1.0 \mathrm{fm}^{-1}$ in momentum space) cannot resolve the necessary degrees of freedom. Since the Compton length of the pion is approximately $0.7 \mathrm{fm}$, a cutoff $\Lambda=1.0 \mathrm{fm}^{-1}$, which corresponds to $1.0 \mathrm{fm}$ in coordinate space, is too small to resolve the exchange of a pion. Although the resulting renormalized interaction $V_{\text {lowk }}$ with $\lambda=1.0 \mathrm{fm}^{-1}$ may not contain an appropriate tensor force for shell-model calculations, we include its result in Fig. 2 and subsequent similar figures for the sake of completeness.

We now present the results for the cutoff values $\Lambda=$ 1.0,2.1 and $5.0 \mathrm{fm}^{-1}$ in Fig. 22 The matrix elements are calculated using a harmonic oscillator basis with $\hbar \omega=14$ and $11 \mathrm{MeV}$ for the $s d$-shell and the $p f$-shell, respectively. Except for a very low (and thereby unreasonable) cutoff value $\Lambda=1.0 \mathrm{fm}^{-1}$, one finds, both in the $s d$-shell and the $p f$-shell, that the monopole part of the tensor force of $V_{\text {low }}$ has almost no cutoff dependence, and has almost the same strength as that of the original NN interaction. Thus, within the usual values of the cutoff, we can see that the monopole part of the tensor force fulfills the R-Persistency almost perfectly with respect to the renormalization of the short-range part of the NN interaction.

We look now into the robustness and the generality of the features discussed above. For this purpose, we consider the relative motion of two interacting nucleons. 

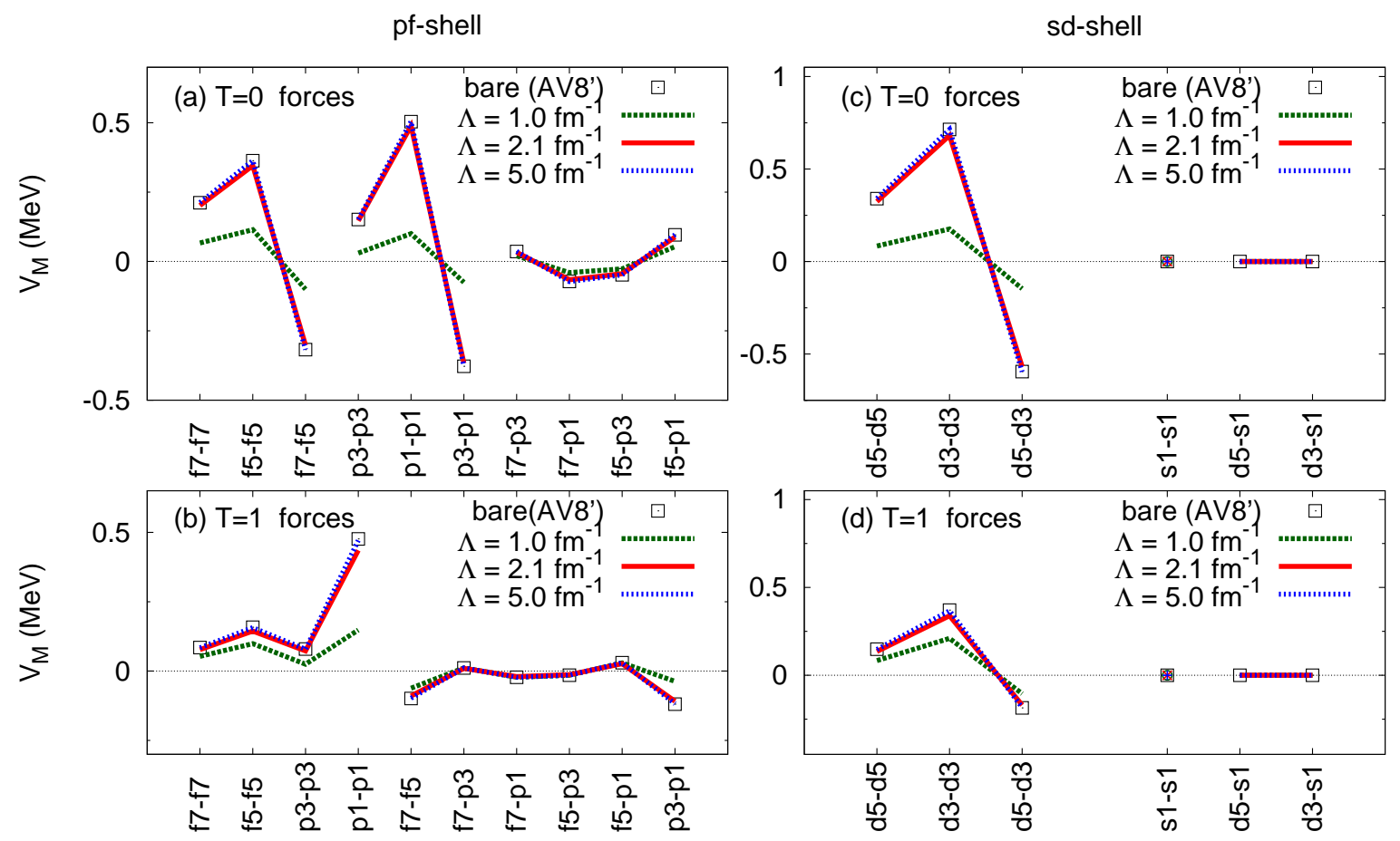

FIG. 2: (color online.) Tensor-force monopole component of low-momentum interaction $V_{\text {low } k}$ as function of the cutoff parameter $\Lambda$ for (a) $T=0$ forces in $p f$-shell, (b) $T=1$ forces in $p f$-shell, (c) $T=0$ forces in $s d$-shell and (d) $T=1$ forces in $s d$-shell. The cutoff parameter $\Lambda$ of $V_{\text {lowk }}$ varies from $1.0 \mathrm{fm}^{-1}$ to $5.0 \mathrm{fm}^{-1}$.

The orbital angular momentum of the relative motion can be $L=0(S), 1(P), 2(D)$, etc. If the tensor force is acting between two states, there is no coupling between two $S$ states, because the relative coordinate operator in the tensor force is of rank 2. The $S$-to- $S$ coupling is thus zero. This results in strongly suppressed contributions to the tensor force from the short-range part of the relativemotion wave function, since a good fraction of the shortrange repulsion stems from $S$ waves. Partial waves higher than $S$ waves carry also a centrifugal barrier component which results in smaller short-range contribution to the tensor force relative to $S$ waves. Thus, changes of the potential at short distances do not affect matrix elements of the tensor force for low momentum states. This seems to be the basic reason why the tensor force remains almost the same throughout the renormalization procedure. In other words, there is a sound reason to expect the RPersistency for the tensor force regarding the treatment of the short-range correlation. On the other hand, the present argument may not be applied to other parts of the nuclear force such as the central force.

The second term $\delta V$ of Eq. (3) is due to the renormalization. It includes for example the central-force component at intermediate inter-nucleon distances, and may affect, in principle, the tensor force as well. The first term, $P_{\Lambda} V_{\mathrm{bare}} P_{\Lambda}$, is equal to the bare tensor force in the limit of $\Lambda \rightarrow \infty$ by definition. In this limit $\delta V$ is zero. Since matrix elements of the tensor force, particularly for low-momentum states, are not affected much by the short-range modification, the effect of the tensorforce component in the first term of Eq. (3) remains the same to a large extent, even with finite $\Lambda$ values, unless it becomes extremely small. The fact that the RPersistency is almost fulfilled in numerical calculations (as we can see in Fig. 21) implies therefore that the second term $\delta V$ results in small contributions to the tensor force, or does not change the long-range part of the tensor force. The origin of the weak tensor force component in $\delta V$ can be understood by the arguments presented in Sec. $\mathrm{V}$ arguments which are based on the close relation between the $V_{\text {low } k}$ renormalization process and contributions from MBPT that represent long-range corrections, as discussed in 8, 16] as well. We shall come back to this point in Sec. V

\section{RENORMALIZATION OF THE CENTRAL FORCE}

Contrary to the tensor force, it can be seen from our numerical studies that the central force does not fulfill the R-Persistency, and is indeed affected strongly by the renormalization procedure due to the short-range part of the NN interaction. This is reflected in a much stronger 
cutoff dependence as well. The central-force monopole part of $\delta V$ in Eq. (3) is thus not small.

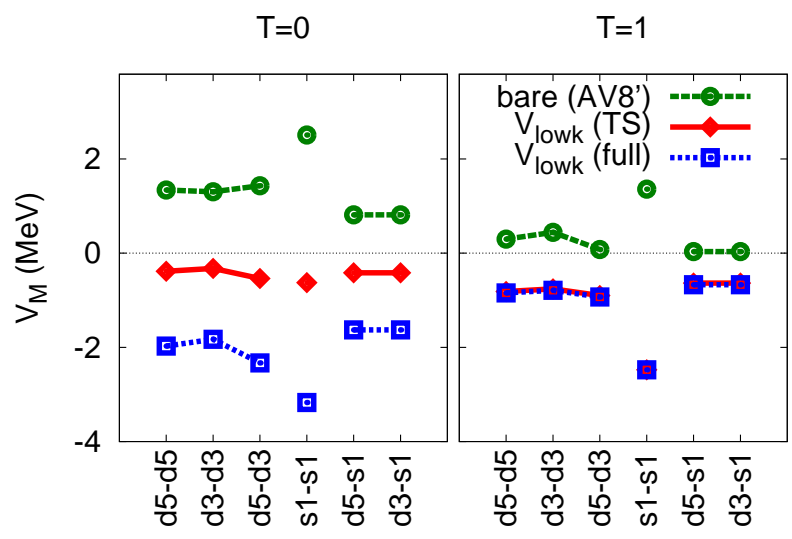

FIG. 3: (color online.) Central-force component of the monopole term of the bare AV8' (bare in the figure), $V_{\text {lowk }}$ (TS) and $V_{\text {lowk }}$ (full) for the $s d$-shell, see text for further details and discussions. The central-force component is obtained using the decomposition of Eq. (7). The effect of the renormalization on the short-range tensor force is also shown. The cutoff value is chosen as $\Lambda=2.1 \mathrm{fm}^{-1}$.

Figure 3 shows the monopole part of the central force of the bare AV8' potential obtained by the decomposition of Eq. (7). In this figure we show also the corresponding central-force monopole component using the $V_{\text {low } k}$ renormalized interaction originating from the AV8' potential, labelled by "full" in the above figure. We show also results where the tensor force component has been subtracted from the bare NN interaction in the renormalization procedure, labelled by "TS" in the figure. What we can see in Fig. Bis the effect of the renormalization due to the short-range part of the bare realistic $\mathrm{NN}$ interaction. The difference between "bare AV8'" and "Vlowk (TS)" lies mainly in the renormalization due to the short-range part of the central force, as the tensor force is subtracted in " $V_{\text {lowk }}(\mathrm{TS})$ ". On the other hand, the difference between "Vlowk (TS)" and "Vlowk (full)" comes solely from the renormalization due to the short-range part of the tensor force.

In the $T=0$ channel, the effect of the renormalization procedure on the short-range part of the tensor force is comparable to that of the central force, while in the $T=1$ channel this effect is almost negligible. This is a quite remarkable feature. Let us discuss this feature in some detail by considering the Schrödinger equation for the deuteron. The deuteron has isospin $T=0, \operatorname{spin} S=1$, orbital momentum $L=0$ ( $S$-wave) and total angular momentum $J=1$. There is a small admixture of $D$ waves as well, leading to the following coupled differential equations for the deuteron

$$
\begin{gathered}
-\frac{\hbar^{2}}{M} \frac{\mathrm{d}^{2} u(r)}{\mathrm{d} r^{2}}+V_{\mathrm{C}} u(r)+\sqrt{8} V_{\mathrm{T}} w(r)=E_{d} u(r), \\
-\frac{\hbar^{2}}{M} \frac{\mathrm{d}^{2} w(r)}{\mathrm{d} r^{2}}+\left(\frac{6 \hbar^{2}}{M r^{2}}+V_{\mathrm{C}}-2 V_{\mathrm{T}}-3 V_{\mathrm{LS}}\right) w(r) \\
+\sqrt{8} V_{\mathrm{T}} u(r)=E_{d} w(r),
\end{gathered}
$$

where $u(r)$ and $w(r)$ are the radial wavefunctions of the $S$-wave and the $D$-wave, respectively. The potentials $V_{\mathrm{C}}, V_{\mathrm{LS}}$ and $V_{\mathrm{T}}$ are the central, spin-orbit and tensor forces, respectively. Knowing the solution of Eq. (8), we can integrate out the $D$-wave degrees of freedom and obtain the following effective central force

$$
\begin{array}{r}
V_{\mathrm{eff}}\left(r ;{ }^{3} S_{1}\right)=V_{\mathrm{C}}\left(r ;{ }^{3} S_{1}\right)+\Delta V_{\mathrm{eff}}\left(r ;{ }^{3} S_{1}\right), \\
\Delta V_{\mathrm{eff}}\left(r ;{ }^{3} S_{1}\right) \equiv \sqrt{8} V_{\mathrm{T}}(r) \frac{w(r)}{u(r)} .
\end{array}
$$

The effective central force $\Delta V_{\text {eff }}$ is comparable to $V_{\mathrm{C}}$ in strength and it makes the ${ }^{3} S_{1}$ channel the most attractive one [17, 18]. This effective central force makes the deuteron bound for the ${ }^{3} S_{1}$ channel. We can regard this equation as a special case of Eq. (3). The effective central force comes from a second-order effect due to tensor force, since both the initial and the final state have orbital angular momentum 0 . As a consequence, the effective interaction for the $T=0$ channel is enhanced by the renormalization procedure due to the short-range part of the tensor force. This is however not the case in $T=1$ channel. It reflects the property of the deuteron, which is the only bound two-nucleon system. A similar mechanism may also explain the strong cutoff dependence of the $V_{\text {low } k}$ interaction seen in the $T=0$ channel.

\section{TENSOR FORCE IN EFFECTIVE INTERACTION FOR THE SHELL MODEL}

We discuss here the tensor-force component in the effective interactions for the shell model, using the decomposition of Eq. (77). We have calculated effective interactions for the shell model $\left(V_{\mathrm{eff}}^{\mathrm{SM}}\right)$ using many-body perturbation theory (MBPT) by considering the $\hat{Q}$-box up to second and third order with folded diagrams included as well, starting from a renormalized $V_{\text {lowk }}$ interaction. The cutoff value used in the $V_{\text {lowk }}$ calculation is set to $\Lambda=2.1 \mathrm{fm}^{-1}$ [15]. The model space $(P$-space $)$ is chosen to be the full $s d$-shell or the full $p f$-shell. In the construction of the $V_{\text {low } k}$ interaction, we renormalize the strong short-range repulsion of the NN interaction, and in MBPT we include further effects of truncations of the model space. The $\hat{Q}$-box is calculated by considering valence-linked and connected diagrams with unperturbed single particle energies of the harmonic oscillator. The oscillator energy $\hbar \omega$ is set to be $14 \mathrm{MeV}$ and $11 \mathrm{MeV}$ for the $s d$-shell and the $p f$-shell effective interactions, respectively. Degenerate perturbation theory is employed in constructing the effective interactions. 
Since the $Q$-space is defined as the complement of the $P$-space, intermediate states arising in each diagram should be taken up to infinitely high oscillator shells. In our case, using a low-momentum interaction $V_{\text {low }}$ with $\Lambda=2.1 \mathrm{fm}^{-1}$, full convergence of the monopole part of $V_{\text {eff }}$ is obtained with approximately $8-10 \hbar \omega$ excitations in each diagram which makes up the $\hat{Q}$-box.

Figure 4 shows the monopole part of the tensor force of $V_{\text {eff }}^{\mathrm{SM}}$ defined for the $s d$-shell or the $p f$-shell. As a general trend, one can see again that the monopole part of the tensor force of $V_{\text {eff }}^{\mathrm{SM}}$ fulfills our R-Persistency hypothesis to a good extent both in the $s d$-shell and in the $p f$-shell. Since the first order $\hat{Q}$-box is just the $V_{\text {low } k}$ interaction, the results mean that the monopole part of the tensor force is dominated by the first-order term in the $\hat{Q}$-box and the contributions from second or higher-order terms are remarkably small. These results can be understood by considering the specific angular momentum structure of the tensor force, which is a scalar product of two rank 2 tensors in spin and coordinate spaces. In a perturbative correction to second or higher-order, such a complicated structure is smeared out and the resulting interaction consists mainly of a central force contribution. Therefore, as for the tensor-force component in the monopole interaction, it is the first-order contribution which is the dominant one.

To elucidate why higher-order terms in many-body perturbation theory are small, we consider as an example a contribution from second order in the interaction, by far the largest higher-order term.

The Hamiltonian causing the present second-order perturbation can be written as

$$
H_{1}=\sum_{p=0,1,2} w_{p}\left(U^{(p)} \cdot X^{(p)}\right)
$$

where $w_{p}$ represents an interaction strength, $U^{(p)}$ and $X^{(p)}$ are operators of rank $p$ in spin space and coordinate space, respectively. A contribution from second-order in perturbation theory to a state $\phi$ can then be written as

$$
\eta(\phi)=-\sum_{j} \frac{\left\langle\phi\left|H_{1}\right| \psi_{j}\right\rangle\left\langle\psi_{j}\left|H_{1}\right| \phi\right\rangle}{\Delta E_{j}}
$$

where $\psi_{j}$ defines an intermediate state with energy denominator $\Delta E_{j}$. The summation is done over all intermediate states $\psi_{j}$. As far as $\psi_{j}$ varies in this summation, within a fixed configuration with respect to harmonicoscillator (HO) shells, $\Delta E_{j}$ remains constant due to the degeneracy of single-particle energies in a given $\mathrm{HO}$ shell. We mention that the usage of non-degenerate perturbation theory yields only small changes.

Such a configuration for a given HO shell is denoted by $S$. As $\Delta E_{j}$ is a constant within a fixed shell $S$, we label it as $\Delta E_{S}$. Note that $S$ corresponds to a part of the $Q$-space, while $\phi$ is in the $P$-space. The term $\eta(\phi)$ can then be decomposed into contributions from individual $S$ 's as

$$
\eta(\phi)=-\sum_{S} \frac{\zeta(\phi, S)}{\Delta E_{S}}
$$

where

$$
\zeta(\phi, S)=\sum_{j \in S}\left\langle\phi\left|H_{1}\right| \psi_{j}\right\rangle\left\langle\psi_{j}\left|H_{1}\right| \phi\right\rangle
$$

For a given $S$, all $\psi_{j}$ s are included, and the summation can be replaced by the closure relation as

$$
\zeta(\phi, S)=\left\langle\phi\left|\left\{H_{1} H_{1}\right\}_{S}\right| \phi\right\rangle
$$

where the parentheses \{\}$_{S}$ are introduced to indicate that the second $H_{1}$ changes $\phi$ to an $S$-configuration state in the $Q$-space and the first $H_{1}$ moves it back to state $\phi$ in the $P$-space. In other words, $H_{1} H_{1}$ in this equation cannot be a simple product, but a certain contraction is needed as we shall show soon.

By utilizing Eq. (10), we obtain

$$
\begin{aligned}
\left\{H_{1} H_{1}\right\}_{S} & =\sum_{p_{1}, p_{2}} w_{p_{1}} w_{p_{2}}\left\{\left(U^{\left(p_{1}\right)} \cdot X^{\left(p_{1}\right)}\right)\left(U^{\left(p_{2}\right)} \cdot X^{\left(p_{2}\right)}\right)\right\}_{S} \\
& =\sum_{k=0,1,2}(2 k+1)\left(\sum_{p_{1}, p_{2}} w_{p_{1}} w_{p_{2}}\left\{\begin{array}{ccc}
p_{1} & p_{2} & k \\
p_{1} & p_{2} & k \\
0 & 0 & 0
\end{array}\right\}\left\{\left[\left[U^{\left(p_{1}\right)} \times U^{\left(p_{2}\right)}\right]^{(k)} \times\left[X^{\left(p_{1}\right)} \times X^{\left(p_{2}\right)}\right]^{(k)}\right]^{(0)}\right\}_{S}\right),
\end{aligned}
$$

where the terms in curly brackets are $9 j$ symbols and $k$ implies the rank of the recoupling. The operator $\left\{\left[U^{\left(p_{1}\right)} \times U^{\left(p_{2}\right)}\right]^{(k)}\right\}_{S}$ acts in the $P$-space as a rank- $k$ twobody operator in spin space, while $\left\{\left[X^{\left(p_{1}\right)} \times X^{\left(p_{2}\right)}\right]^{(k)}\right\}_{S}$ acts as a rank- $k$ two-body operator in coordinate space. Because the contraction due to the elimination of the $Q$ space does not affect the angular momentum properties, the variable $k=0,1,2$ represents induced central, spin- 

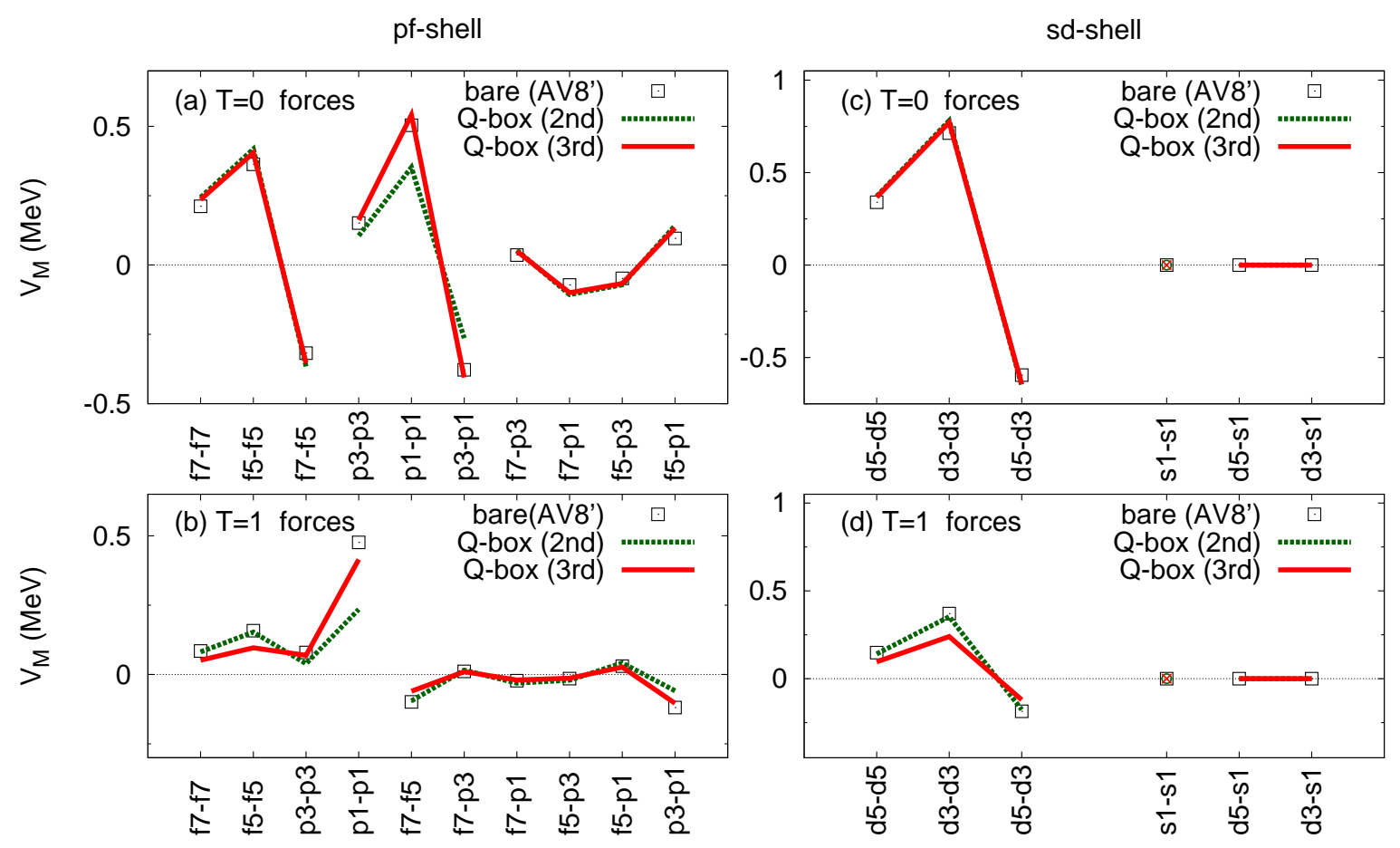

FIG. 4: (color online.) The tensor force monopole component of the effective interaction for the shell model obtained by the $\hat{Q}$-box expansion to second and third order in the interaction, starting from $V_{\text {lowk}}\left(\Lambda=2.1 \mathrm{fm}^{-1}\right)$. The tensor-force component is obtained using the decomposition of Eq. (7). (a) $T=0$ forces in $p f$-shell, (b) $T=1$ forces in $p f$-shell, (c) $T=0$ forces in $s d$-shell and (d) $T=1$ forces in $s d$-shell.

orbit and tensor forces in the $P$-space, respectively.

Since we are mainly interested in the tensor component, we focus on the case of $k=2$, with the obvious restriction $p_{1}+p_{2} \geq 2$. Since the above $9 j$ symbols is proportional to $1 / \sqrt{\left(2 p_{1}+1\right)\left(2 p_{2}+1\right)}$, it is easy to convince oneself that the central force component receives the largest contribution from the $9 j$ symbol. Furthermore, for our analyses it is important to keep in mind that the expectation value of the central component is the largest in absolute value, the tensor component the second largest and the spin-orbit term gives rise to the smallest contribution to the renormalized $V_{\text {low } k}$ interaction.

From these considerations, for $k=2$ the largest contribution comes from the combination $p_{1}=0, p_{2}=2$ or $p_{1}=2, p_{2}=0$ in Eq. (10), that is either a central-tensor or a tensor-central combination. Let us now discuss this case. We assume without loss of generality that the tensor component of $H_{1}$ acts on the ket state of the matrix element being considered. While the central component of $H_{1}$ acts afterward on this state, we can also consider that this central force acts to the left on the bra state. We then take the overlap between these two states by considering one by the tensor on the ket side, and the other by the central on the bra side. These two states are sumrule states for the two forces within the $S$-configuration space. As the central force and the tensor force are very different in nature, such sum-rule states are very different from each other in general, leading to a very small overlap. This is the main reason why the combination of the central force and the tensor force produces small contributions.

This argument does not hold for the case where the tensor component of $H_{1}$ acts twice in the term to secondorder in perturbation theory. However, due to the angular momentum coupling, the product of two tensor forces $\left(p_{1}=p_{2}=2\right.$ in Eq. (10) ) yield small contributions to the $k=2$ terms of Eq. (10). For higher orders, other tensor-force components may show up, but there is no mechanism to enhance their contributions.

The small contribution of the tensor force in MBPT can be viewed to be reasonable also under the following intuitive picture: after multiple actions of the forces, the spin dependence is smeared out, and only the distance between two interacting nucleons becomes the primary factor to the whole processes. This results in the dominance of the induced effective interaction by the central components and yields only a minor change in the tensor component.

It is instructive to study in more detail the contributions to second-order in perturbation theory. To do so, we single out the by far largest second-order term, namely 
the so-called core-polarization term, depicted as diagram (c) in Fig. 1, For the core-polarization diagram we can show that the contribution to the tensor force vanishes by simple angular momentum algebra arguments. The contribution to a specific core-polarization matrix element can then be written as

$$
\begin{aligned}
\left\langle a m_{a} b m_{b}\left|V_{T}^{\mathrm{cp}-\mathrm{eff}}\right| c m_{c} d m_{d}\right\rangle= & \sum_{p, m_{p}, h, m_{h}}\left\langle a m_{a} p m_{p}\left|V_{C}\right| c m_{c} h m_{h}\right\rangle\left\langle h m_{h} b m_{b}\left|V_{T}\right| p m_{p} d m_{d}\right\rangle / \Delta E \\
= & \sum_{n_{p}, l_{p}, n_{h}, l_{h}}\left(\sum_{j_{p}, m_{p}, j_{h}, m_{h}}\left\langle a m_{a} p m_{p}\left|V_{C}\right| c m_{c} h m_{h}\right\rangle\left\langle h m_{h} b m_{b}\left|V_{T}\right| p m_{p} d m_{d}\right\rangle\right) / \Delta E_{S} \\
= & \sum_{n_{p}, l_{p}, n_{h}, l_{h}}\left(\sum_{m_{l p}, m_{s p}, m_{l h}, m_{s h}}\left\langle a m_{a} n_{p} l_{p} m_{l p} m_{s p}\left|V_{C}\right| c m_{c} n_{h} l_{h} m_{l h} m_{s h}\right\rangle\right. \\
& \left.\times\left\langle n_{h} l_{h} m_{l h} m_{s h} b m_{b}\left|V_{T}\right| n_{p} l_{p} m_{l p} m_{s p} d m_{d}\right\rangle\right) / \Delta E_{S},
\end{aligned}
$$

where $V_{T}^{\mathrm{cp}-\mathrm{eff}}$ is the induced tensor force, $V_{T}$ and $V_{C}$ are the tensor force and central force components from $H_{1}$, respectively. With a harmonic oscillator basis, the term $\Delta E_{S}$ represents an energy denominator which is constant for a given set of quantum numbers $n_{p}, n_{h}, l_{p}$ and $l_{h}$, as discussed above. Here $a=\left(n_{a}, l_{a}, j_{a}\right)$, and $m_{a}$ denotes magnetic substate of $l_{a}$. Note that the twobody states are not antisymmetrized. The states $p$ and $h$ represent particle and hole states, respectively. In the third line of the equation, only particle and hole states are transformed to the $l s$ coupling scheme. Note that the intermediate states are summed up to fulfill spinsaturation within each $\mathrm{HO}$ major shell.

We can divide the contribution into two different types according to the spin dependence of the central force. One comes from the terms whose central force part $V_{C}$ includes $\sigma \cdot \sigma$ (type I) and the other does not (type II). With our summation tailored to a spin-saturated core or an excluded $Q$-space with all spin-orbit partners, we can prove that type II contributions always vanish because the first factor is diagonal with respect to spin, that is $m_{s p}=m_{s h}$, and the second factor is zero when we sum over spin-saturated contributions. Therefore, only a spin-dependent central force results in non-vanishing contributions to the tensor force for higher-order terms in $V_{\mathrm{eff}}^{\mathrm{SM}}$. Finally, the contribution to the tensor force from the spin-dependent central force is quite small because the spin-dependent central force is generally by far smaller than the spin-independent central force in modern realistic NN potentials.

In conclusion, medium effects produce minor contributions to the tensor-force component, resulting in a tensorforce component that is dominated by the bare NN interaction. Our hypothesis about Renormalization Persistency is fulfilled to a good extent by the tensor force.

Finally, although our analyses has been performed within one major shell only, one should note that this persistency of the tensor-force component via a MBPT renormalization should also hold in for model spaces which span several shells.

\section{TWO-BODY MATRIX ELEMENTS OF THE TENSOR FORCE}

In this section we study further renormalization properties of the tensor force by including higher multipole components.

Figure 5 shows the diagonal and non-diagonal matrix elements of the bare tensor force from the AV8' potential, the renormalized $V_{\text {low } k}$ interaction $\left(\Lambda=2.1 \mathrm{fm}^{-1}\right)$ and $V_{\mathrm{eff}}^{\mathrm{SM}}$ obtained by the $\hat{Q}$-box expansion up to the third order with folded diagrams to infinite order starting from AV8' interaction. This is similar to what was done in Figs. 2 and 4. In this figure, panels (a) to (d) stand for the $s d$-shell matrix elements. The diagonal matrix elements are shown in panels (a) and (b) while the non-diagonal elements are shown in panels (c) and (d). Note that the diagonal matrix elements $\left\langle j_{a} j_{b}|V| j_{a} j_{b}\right\rangle_{J T}$ are specified by the quantum numbers $j_{a}, j_{b}$, and twice the total angular momentum $J$ and total isospin $T$. The non-diagonal matrix elements $\left\langle j_{a} j_{b}|V| j_{c} j_{d}\right\rangle_{J T}$ are specified by $j_{a}, j_{b}, j_{c}, j_{d}, J$ and $T$. The corresponding numbers for the $p f$-shell are shown in panels (e) through (j). In both the $s d$-shell and the $p f$-shell, the patterns are the same for all approaches to the effective interactions and thus the R-Persistency is approximately fulfilled. In particular, for the $V_{\text {lowk }}$ renormalization procedure, we can hardly see any difference between the bare tensor force and the tensor force in the effective interaction $V_{\text {low }}$. For the diagonal matrix elements, we can see small differences between the final $\hat{Q}$-box and the bare tensor force, however, it does not contradict the results with respect to the monopole component discussed above, mainly because only matrix elements with small values of the total angular momentum display sizable differences. Since the monopole terms are weighted by $2 J+1$, matrix elements with larger values of the total angular momentum $J$ carry 
a much larger weight in Eq. (10). In non-diagonal matrix elements, we see somewhat larger differences. Their role in shell-model calculations needs to be investigated further. A spin-tensor analysis along these lines was made recently by Smirnova et al [19].

\section{ANALYSIS OF OTHER INTERACTION MODELS}

In the previous sections, we calculated effective interactions starting from the AV8' interaction, using a renormalized interaction and many-body perturbation theory. We found that the R-Persistency of the tensor force holds for all these renormalization procedures. An obvious question is whether or not the R-Persistency holds for other interaction models as well. In this section we address this issue as well.

We employ here another frequently used realistic interaction, $\chi \mathrm{N}^{3} \mathrm{LO}$, as an example [11]. The $\chi \mathrm{N}^{3} \mathrm{LO}$ interaction has a relatively smaller coupling between low-momentum and high-momentum modes compared with the AV8' potential. In Fig. 6 we show the monopole part of the tensor force of the $\chi \mathrm{N}^{3} \mathrm{LO}$ bare potential and $V_{\text {low } k} \mathrm{~s}$ with several cutoff parameters $\Lambda\left(1.0 \mathrm{fm}^{-1}, 2.1 \mathrm{fm}^{-1}\right.$ and $\left.5.0 \mathrm{fm}^{-1}\right)$. These results should be compared with the corresponding ones obtained with the AV8' interaction shown in Fig. 22 Figure 7 shows the tensor-force monopole of $V_{\text {eff }}^{\mathrm{SM}}$, corresponding to Fig. 4 for the AV8' interaction, starting from the $\chi \mathrm{N}^{3} \mathrm{LO}$ interaction. Finally, Fig. 8 shows the multipole components of effective interactions, corresponding to Fig. 5 for the AV8' interaction.

In all the figures, we can conclude that all the features we discussed for the AV8' interaction pertain to the $\chi \mathrm{N}^{3} \mathrm{LO}$ interaction model as well.

\section{CONCLUSION}

In this work we have presented a detailed analysis of various contributions to the nuclear tensor force as function of different renormalization procedures, starting with state-of-the-art nucleon-nucleon (NN) interactions and ending up with effective interactions for the nuclear shell model. The monopole part of the tensor force is weakly or barely affected by various renormalization procedures, which in our case are represented by a renormalization of the bare interaction and many-body pertur- bation theory in order to obtain an effective shell-model interaction. This has lead us to introduce the concept of renormalization persistency (R-Persistency) in the study of effective interactions. We studied the R-persistency of both renormalization procedures and showed via numerical studies, their intuitive general explanations and a detailed algebraic analysis of core-polarization terms in perturbation theory, that this is a very robust process. We have also shown that the R-Persistency holds for twobody matrix elements including higher multipole components of the tensor force, although the deviation increases somewhat if multipole components are included in the comparison. We conclude that the two renormalization steps (one for short-range correlation and the other for inmedium effects) do not affect much either the monopole nor the multipole components of the tensor force, apart from slight differences between them. Results obtained with two different interactions (AV8' and $\chi \mathrm{N}^{3} \mathrm{LO}$ ) lead us to the same conclusion, suggesting that the R-Persistency of the tensor force for low-momentum states is a robust feature. This applies also to other interaction models than those studied here.

The short-range part of the tensor force enters the renormalization of the central force, in particular in the $T=0$ channel, producing on average an increased attraction. Since the modification of the tensor force appears to be small, the central force carries most of the renormalization effects beyond first order in perturbation theory.

Because the R-Persistency of the tensor force in effective interactions is a robust feature, it may give a simple and concrete starting point for examining and constructing effective interactions, especially phenomenological ones [4]. In particular, since the tensor force plays a significant role in the shell evolution for nuclear systems with either large neutron/proton or proton/neutron ratios, one can extract simple physics messages from complicated many-body systems.

\section{Acknowledgments}

We are very grateful to Professors R. Okamoto and H. Feldmeier for valuable discussions. This work is supported in part by Grant-in-Aid for Scientific Research (A) 20244022 and also by Grant-in-Aid for JSPS Fellows (No. 228635), and by the JSPS Core to Core program "International Research Network for Exotic Femto Systems" (EFES).
[1] R. B. Wiringa, V. G. J. Stoks, and R. Schiavilla, Phys. Rev. C 51, 38 (1995).

[2] R. B. Wiringa and S. C. Pieper, Phys. Rev. Lett. 89, 182501 (2002).

[3] T. Otsuka, T. Suzuki, R. Fujimoto, H. Grawe, and Y. Akaishi, Phys. Rev. Lett. 95, 232502 (2005).
[4] T. Otsuka, T. Suzuki, M. Honma, Y. Utsuno, N. Tsunoda, K. Tsukiyama, and M. Hjorth-Jensen, Phys. Rev. Lett. 104, 012501 (2010).

[5] R. Bansal and J. French, Physics Letters 11, 145 (1964).

[6] A. Poves and A. Zuker, Physics Reports 70, 235 (1981).

[7] S. Bogner, T. T. S. Kuo, L. Coraggio, A. Covello, and 
N. Itaco, Phys. Rev. C 65, 051301 (2002).

[8] S. K. Bogner, T. T. S. Kuo, and A. Schwenk, Physics Reports 386, 1 (2003).

[9] T. Kuo and E. Osnes, Lecture Notes in Physics 364, 1 (1990).

[10] M. Hjorth-Jensen, T. T. S. Kuo, and E. Osnes, Physics Reports 261, 125 (1995).

[11] E. Epelbaum, H.-W. Hammer, and U.-G. Meißner, Rev. Mod. Phys. 81, 1773 (2009).

[12] M. W. Kirson, Physics Letters B 47, 110 (1973).

[13] E. Osnes and D. Strottman, Phys. Rev. C 45, 662 (1992).

[14] B. A. Brown, W. A. Richter, R. E. Julies, and B. H. Wildenthal, Annals of Physics 182, 191 (1988).

[15] A. Nogga, S. K. Bogner, and A. Schwenk, Phys. Rev. C
70, 061002 (2004).

[16] S. K. Bogner, A. Schwenk, T. T. S. Kuo, and G. E. Brown, arXiv nucl-th (2001).

[17] A. Umeya and K. Muto, Phys. Rev. C 74, 034330 (2006).

[18] R. Tamagaki and W. Watari, Progress of Theoretical Physics Supplement 39, 23 (1967).

[19] N. A. Smirnova, B. Bally, K. Heyde, F. Nowacki, and K. Sieja, Physics Letters B 686, 109 (2010).

[20] For the $j=j^{\prime}$ case, the definition is slightly different.

[21] A short version of the present results was included in [4], while more substantial, deeper and wider discussions are given in this paper. 

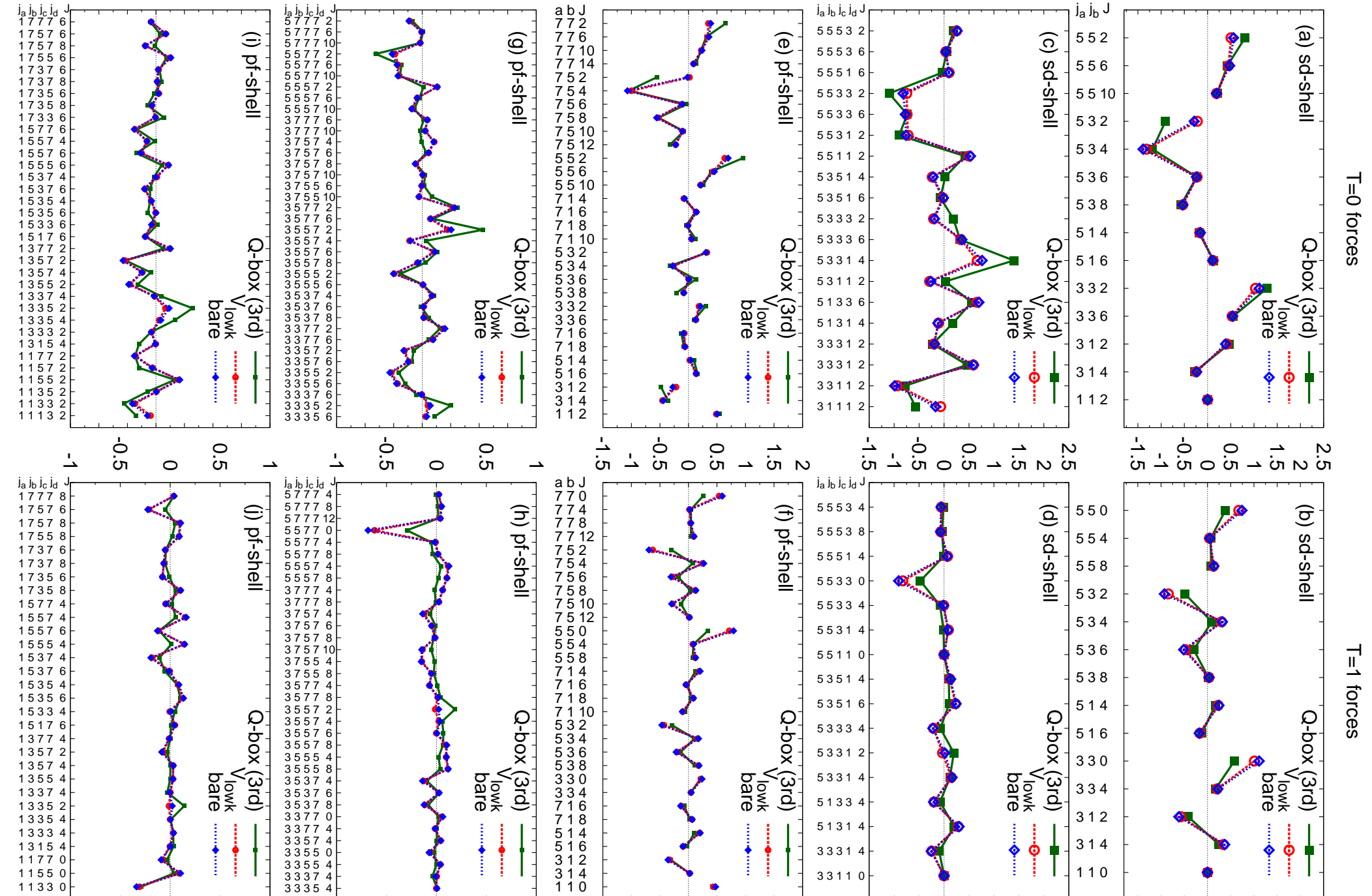

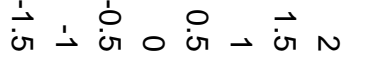

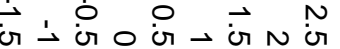

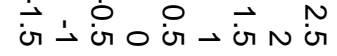
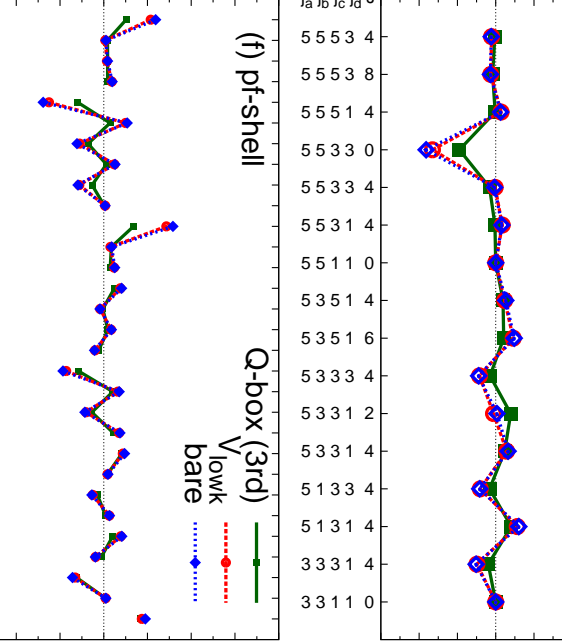

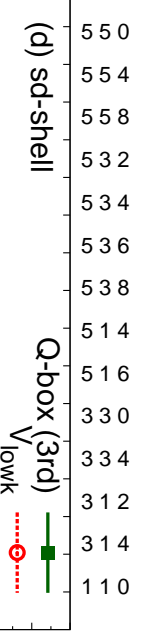

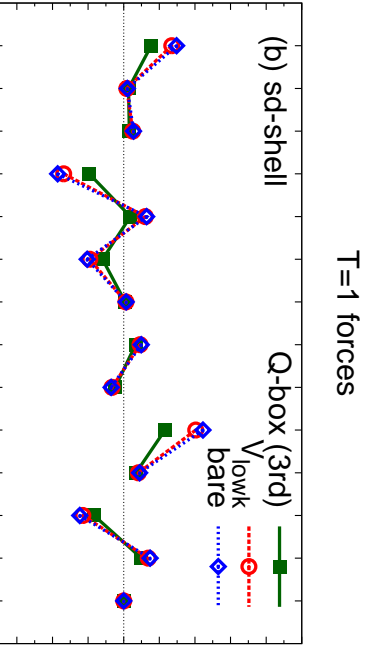


pf-shell
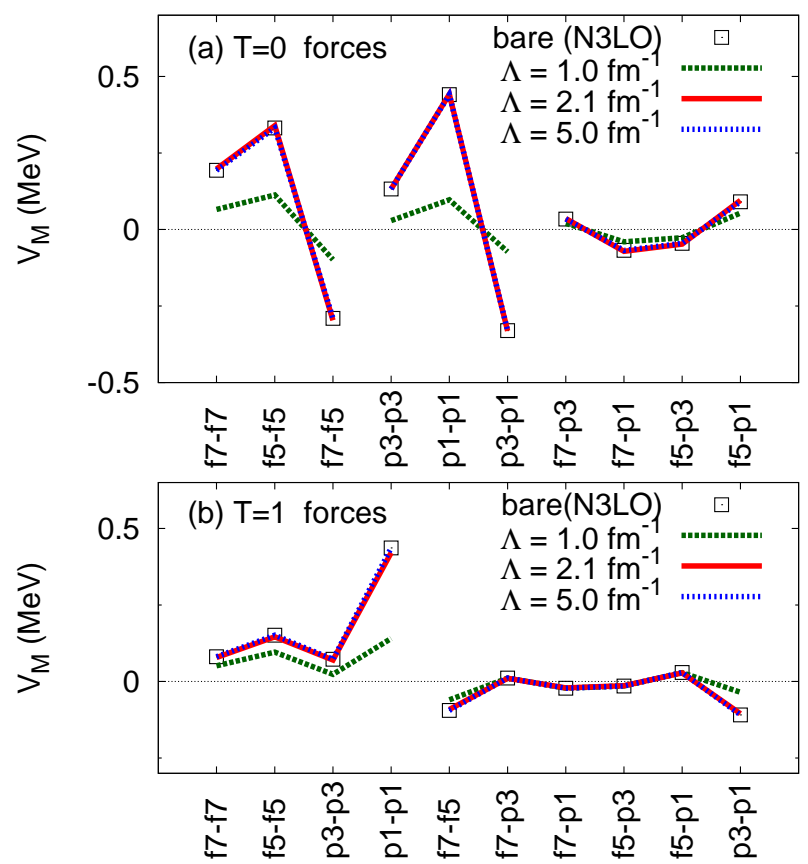

sd-shell
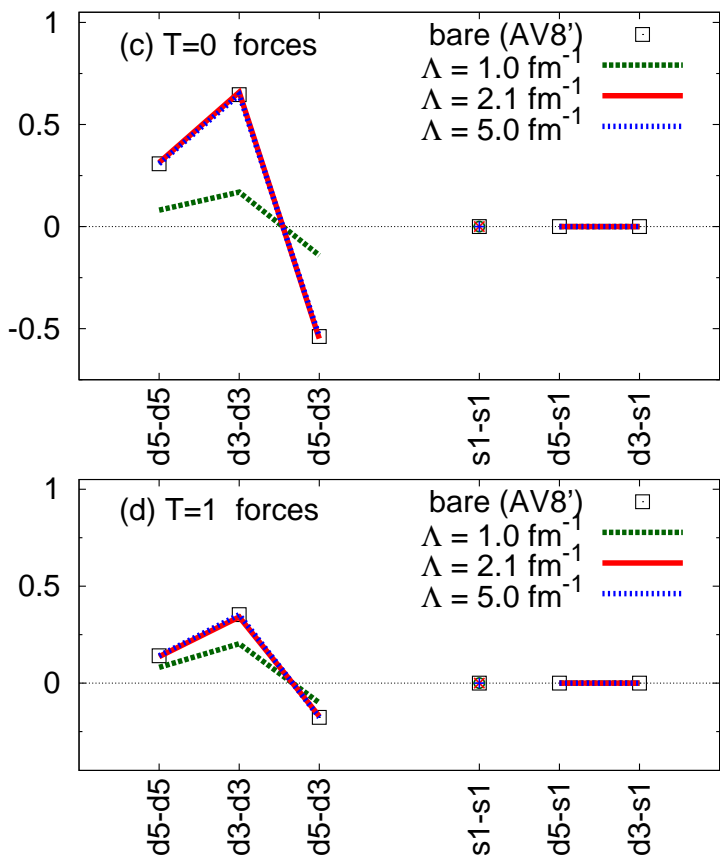

FIG. 6: (color online.) Tensor-force monopole of $V_{\text {lowk }}$ starting from the $\chi \mathrm{N}^{3} \mathrm{LO}$ interaction with the same notation as in Fig. 22
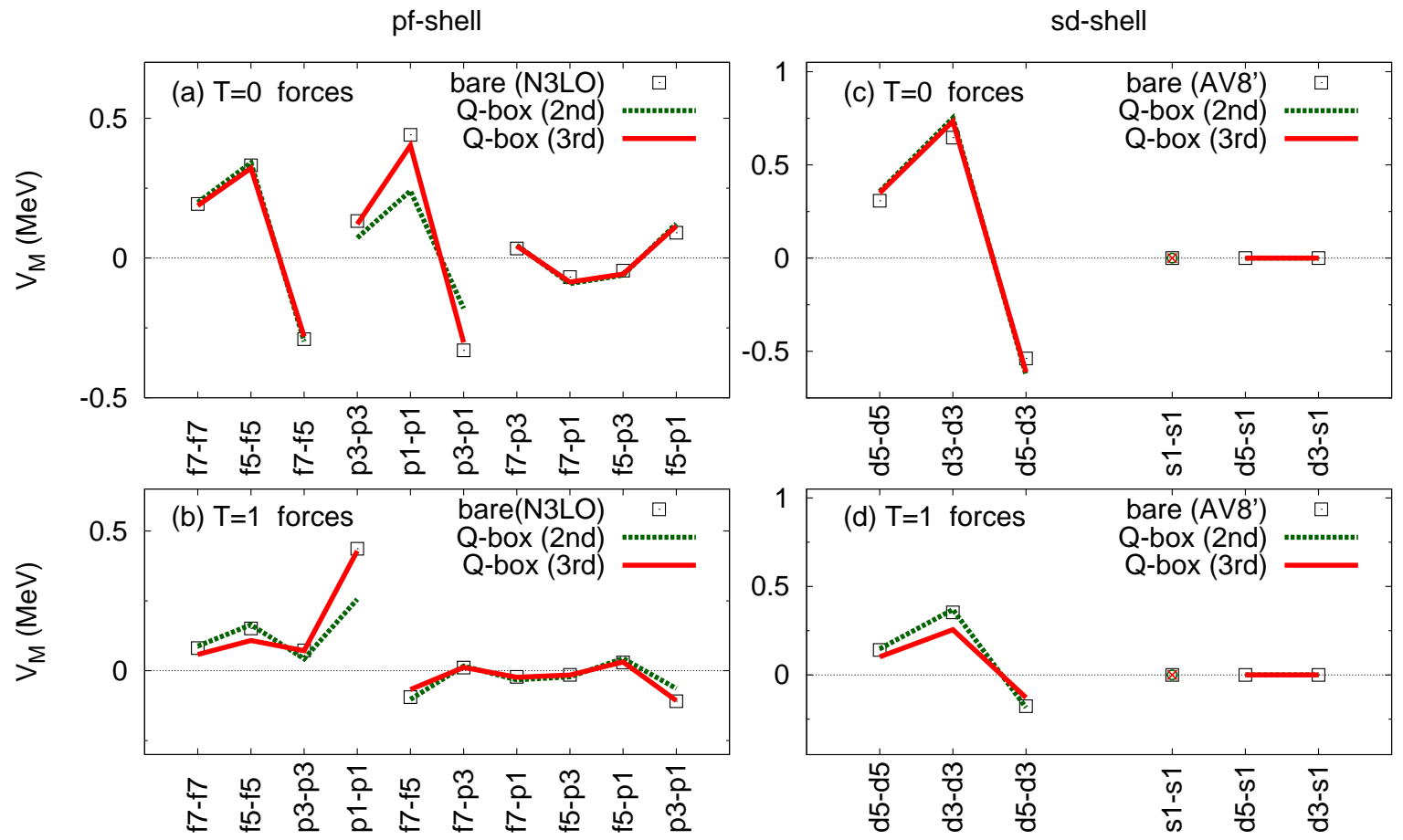

FIG. 7: (color online.) Tensor-force monopole of $V_{\text {eff }}^{\mathrm{SM}}$ starting from the $\chi \mathrm{N}^{3} \mathrm{LO}$ with the same notation as in Fig. [4. 
